\title{
Comparison of five staging systems in predicting the survival rate of patients with hepatocellular carcinoma undergoing trans-arterial chemoembolization therapy
}

\author{
ZHAN-HONG CHEN ${ }^{1,2^{*}}$, YING-FEN HONG ${ }^{1 *}$, XIANGWEI CHEN ${ }^{1 *}$, JIE CHEN $^{1}$, QU LIN ${ }^{1}$, \\ JINXIANG LIN ${ }^{1}$, XING LI ${ }^{1}$, JING-YUN WEN ${ }^{1}$, DAN-YUN RUAN ${ }^{1}$, MIN DONG $^{1}$, LI WEI ${ }^{1}$, TIAN-TIAN WANG ${ }^{1}$, \\ ZE-XIAO LIN ${ }^{1}$, XIAO-KUN MA ${ }^{1}$, DONG-HAO WU ${ }^{1}$, XIANG-YUAN WU ${ }^{1}$ and RUIHUA XU ${ }^{2}$ \\ ${ }^{1}$ Department of Medical Oncology and Guangdong Key Laboratory of Liver Disease, \\ The Third Affiliated Hospital of Sun Yat-Sen University, Guangzhou, Guangdong 510630; ${ }^{2}$ Department of \\ Medical Oncology of Sun Yat-Sen University Cancer Center, State Key Laboratory of Oncology in South China, \\ Collaborative Innovation Center for Cancer Medicine, Guangzhou, Guangdong 510060, P.R. China
}

Received June 19, 2017; Accepted October 13, 2017

DOI: 10.3892/ol.2017.7419

\begin{abstract}
The majority of patients with unresectable hepatocellular carcinoma (HCC) undergo trans-arterial chemoembolization (TACE). However, the prognosis of HCC remains poor. In the present study, five staging systems were compared to predict the survival rate of patients with HCC undergoing TACE treatment. A total of 220 patients with HCC were examined according to the model to estimate survival for hepatocellular carcinoma (MESH), hepatoma arterial embolization prognostic score (HAP), modified HAP (mHAP), performance status combined Japan Integrated Staging system (PSJIS) and tumor-node-metastasis (TNM) staging systems. The endpoints of the study were 3-month survival, 6-month survival, 1-year survival and overall survival (OS) rates. Receiver operating characteristic curve analysis indicated that the area under the curve of MESH, HAP, mHAP, PSJIS and TNM was $0.858,0.728,0.690,0.688$ and 0.699 , respectively, in predicting 3-month survival rates; 0.822, 0.747, 0.720, 0.722 and 0.715 , respectively, in predicting 6-month survival
\end{abstract}

Correspondence to: Professor Xiang-Yuan Wu, Department of Medical Oncology and Guangdong Key Laboratory of Liver Disease, The Third Affiliated Hospital of Sun Yat-Sen University, 600 Tianhe Road, Guangzhou, Guangdong 510630, P.R. China

E-mail:wuxiangy@mail.sysu.edu.cn

Professor Ruihua Xu, Department of Medical Oncology of Sun Yat-Sen University Cancer Center, State Key Laboratory of Oncology in South China, Collaborative Innovation Center for Cancer Medicine, 651 Dongfengdong Road, Guangzhou, Guangdong 510060, P.R. China

E-mail: xurh@sysucc.org.cn

${ }^{*}$ Contributed equally

Key words: hepatocellular carcinoma, hepatitis B, trans-arterial chemoembolization, model to estimate survival for hepatocellular carcinoma, prognosis rates and $0.725,0.664,0.672,0.645$ and 0.654 , respectively, in predicting 1-year survival rates. Discriminatory ability, homogeneity, monotonicity and prognostic stratification ability was evaluated using a likelihood ratio test and Akaike information criterion values among the five staging systems, and revealed that the MESH system was the optimal prognostic staging system for HCC. In conclusion, the results of the present study suggest that the MESH system is the most accurate prognostic staging system of 3-month survival, 6-month survival, 1-year survival and OS rates among the five systems analyzed in patients with HCC who have received TACE treatment.

\section{Introduction}

Hepatocellular carcinoma (HCC) is a common malignancy globally, and its mortality rate ranked fourth in $2015(1,2)$. In total, $60 \%$ of patients with HCC are diagnosed with unresectable HCC, which represents an incurable disease $(3,4)$. Barcelona Clinic Liver Cancer (BCLC) guidelines recommend patients with intermediate-stage $\mathrm{HCC}$ to receive trans-arterial chemoembolization (TACE) as a standard treatment. Two randomized controlled clinical trials demonstrated the benefits of TACE treatment for patients with $\operatorname{HCC}(5,6)$. According to BCLC guidelines, patients with $\mathrm{HCC}$ who present an asymptomatic HCC, lack of portal vein thrombosis or extra-hepatic spread, compensated cirrhosis, and patients classified with Child-Pugh scores $<8$ and a performance status (PS) of 0 , are advised to receive TACE treatment (4). In total, $20 \%$ of patients with $\mathrm{HCC}$ are at an intermediate stage. These patients form a heterogeneous group, owing to discrepancies in tumor burden, serum biomarker, liver function, performance status, etiology, etc. However, BCLC guidelines do not consider all these parameters. The reported survival rates of patients with intermediate-stage HCC varies between 11 and 45 months (7).

Staging systems, including model to estimate survival rates for hepatocellular carcinoma (MESH), hepatoma arterial embolization prognostic score (HAP), modified HAP (mHAP), performance status combined Japan Integrated 
Staging system (PSJIS) and tumor-node-metastasis (TNM), have been developed to determine the optimal treatment for the patients. However, it remains unknown which system is optimal for the prediction of patient survival rates.

In the present study, the performance of five staging systems, i.e., MESH, HAP, mHAP, PSJIS and TNM, was compared in predicting 3-month survival, 6-month survival, 1-year survival and OS survival rates of patients with HBV-associated HCC undergoing TACE. The aim of the present study was to determine the optimal staging system for patients with HCC.

\section{Patients and methods}

Patients. A total of 220 sequential patients with HCC treated with TACE were retrospectively reviewed at the Third Affiliated Hospital of Sun Yat-Sen University (Guangzhou, China) between July 2009 and June 2012. Patient characteristics are provided in Table I. The diagnosis of HCC was confirmed using pathology or magnetic resonance imaging (MRI)/computed tomography (CT) according to the American Association for the Study of Liver Diseases guidelines (3). All participants studied were patients with HBV-associated HCC and received TACE following multidisciplinary team discussion. Patients with advanced HCC classified as Child-Pugh grade A or B and with a performance status (PS) of 0 to 2, platelet count $\geq 30 \times 10^{9}$ cells $/ 1$ and hemoglobin level $\geq 60 \mathrm{~g} / \mathrm{l}$, were eligible for enrollment. However, patients were excluded if a second type of cancer and/or intractable comorbid medical illness existed. Patients classified as Child-Pugh grade $\mathrm{C}$ were also excluded.

Data collection. The Institutional Review Board of the Third Affiliated Hospital of Sun Yat-Sen University reviewed and approved the present study. Prior to enrollment, all participants provided written informed consent for data sharing.

A range of demographic data were collected including risk factors, blood results, imaging and therapy data. Collected data also included sex, age, date of diagnosis, date of mortality or last follow-up date. The clinical records of the patients were retrospectively assessed. Tumor characteristics, including tumor size and extension, vascular invasion and lymph node metastases, were assessed using CT or MRI. Routine blood tests, liver function and coagulation tests were also conducted.

Staging. Baseline data were collected to classify patients according to MESH, HAP, mHAP, PSJIS and TNM systems. All eligible patients were classified by MESH, HAP, mHAP, PSJIS, TNM and BCLC in the first diagnosis, and $91.8 \%$ of patients with HCC were classified according to BCLC-C. Patients with a PS of 2 were also the classified according to BCLC-C at first diagnosis. A baseline evaluation that included laboratory studies, imaging studies (CT or MRI) and clinical examination was performed. Data were collected at the time the patients were diagnosed with advanced HBV-associated HCC. Survival times were defined as the time from first TACE treatment until mortality or last follow-up. Patients who lacked the required data or who were lost to follow-up within 3 months of diagnosis were excluded from the present study.

Statistical analysis. The primary endpoints of the present study were 3 -month survival and OS. The secondary
Table I. Baseline demographic and clinical characteristics of patients.

\begin{tabular}{|c|c|}
\hline Characteristic & Patients \\
\hline Total patients, n (\%) & $220(100)$ \\
\hline \multicolumn{2}{|l|}{ Sex, n $(\%)$} \\
\hline Male & $200(90.90)$ \\
\hline Female & $20(9.10)$ \\
\hline Median age, years (range) & $52.5(11-84)$ \\
\hline \multicolumn{2}{|l|}{ Etiology, $\mathrm{n}(\%)$} \\
\hline HBsAg & $220(100)$ \\
\hline \multicolumn{2}{|l|}{ Tumor size, n (\%) } \\
\hline$<2 \mathrm{~cm}$ & $20(9.1)$ \\
\hline $2-5 \mathrm{~cm}$ & $62(28.2)$ \\
\hline$>5 \mathrm{~cm}$ & $138(62.7)$ \\
\hline Ascites, n (\%) & $55(25.0)$ \\
\hline Portal vein invasion (segmental), $\mathrm{n}(\%)$ & $102(46.4)$ \\
\hline Extrahepatic spread & $21(9.5)$ \\
\hline $\begin{array}{l}\text { White blood cell count, } x 10^{9} \text { cells/ } 1 \\
\text { (range) }\end{array}$ & $5.90(1.54-20.6)$ \\
\hline$\alpha$-fetoprotein, $\mathrm{ng} / \mathrm{ml}$ (range) & $503.47(1-1210)$ \\
\hline Albumin, g/l (range) & $38.06(22.0-53.3)$ \\
\hline Creatinine, $\mu \mathrm{mol} / \mathrm{l}$ (range) & $73.67(41.0-160.1)$ \\
\hline Alkaline phosphatase, U/l (range) & $139.90(44-1048)$ \\
\hline Platelets, $\times 10^{9}$ cells $/ 1$ (range) & $161.31(31-520)$ \\
\hline Hemoglobin, g/l (range) & $130.72(60-190)$ \\
\hline Fibrinogen, $\mathrm{g} / \mathrm{l}$ (range) & $3.48(1.26-9.39)$ \\
\hline Total bilirubin, $\mu \mathrm{mol} / \mathrm{l}$ (range) & $21.28(4.7-109.8)$ \\
\hline AST, IU/l (range) & $82.28(12-931)$ \\
\hline$\gamma$-glutamyltransferase, $\mathrm{U} / \mathrm{l}$ (range) & $166.50(17-1136)$ \\
\hline Blood urea nitrogen, $\mathrm{mmol} / \mathrm{l}$ (range) & $5.24(2.08-12.85)$ \\
\hline PT, sec (range) & $14.18(11.2-24.3)$ \\
\hline INR (range) & $1.11(0.84-2.21)$ \\
\hline 90-day survival rate, $\mathrm{n}(\%)$ & $194(88.18)$ \\
\hline 6-month survival rate, n (\%) & $166(75.45)$ \\
\hline 1-year survival rate, $\mathrm{n}(\%)$ & $132(60.00)$ \\
\hline 2-year survival rate, n (\%) & $108(49.09)$ \\
\hline 5-year survival rate, $\mathrm{n}(\%)$ & $18(8.18)$ \\
\hline \multicolumn{2}{|l|}{ TNM 7 th edition, $\mathrm{n}(\%)$} \\
\hline I & $14(6.4)$ \\
\hline II & $52(23.6)$ \\
\hline III & $38(17.3)$ \\
\hline IV & $116(52.7)$ \\
\hline \multicolumn{2}{|l|}{ Child-Pugh class, n (\%) } \\
\hline A & $153(69.5)$ \\
\hline $\mathrm{B}$ & $67(30.5)$ \\
\hline \multicolumn{2}{|l|}{ BCLC, n (\%) } \\
\hline $\mathrm{A}$ & $6(2.7)$ \\
\hline $\mathrm{B}$ & $12(5.5)$ \\
\hline $\mathrm{C}$ & $202(91.8)$ \\
\hline \multicolumn{2}{|l|}{ MESH, n (\%) } \\
\hline 0 & $5(2.3)$ \\
\hline 1 & $27(12.3)$ \\
\hline 2 & $43(19.5)$ \\
\hline 3 & $60(27.3)$ \\
\hline 4 & $53(24.1)$ \\
\hline 5 & $31(14.1)$ \\
\hline 6 & $1(0.5)$ \\
\hline
\end{tabular}


Table I. Continued.

\begin{tabular}{lc}
\hline Characteristic & Patients \\
\hline HAP, n (\%) & \\
A & $26(11.8)$ \\
B & $59(26.8)$ \\
C & $76(34.5)$ \\
D & $59(26.8)$ \\
mHAP, n (\%) & \\
A & \\
B & $50(22.7)$ \\
C & $74(33.6)$ \\
D & $74(33.6)$ \\
JIS, n $(\%)$ & $22(10.0)$ \\
0 & \\
1 & $6(2.7)$ \\
2 & $52(23.6)$ \\
3 & $81(36.8)$ \\
4 & $56(25.5)$ \\
5 & $22(10.0)$ \\
PSJIS, n $(\%)$ & $3(1.4)$ \\
0 & \\
1 & $1(0.5)$ \\
2 & $12(5.5)$ \\
3 & $44(20.0)$ \\
4 & $76(34.5)$ \\
5 & $46(20.9)$ \\
7 & $27(12.3)$ \\
& $12(5.5)$ \\
& $2(0.9)$ \\
\hline
\end{tabular}

AST, aspartate aminotransferase; BCLC, Barcelona Clinic Liver Cancer; HAP, hepatoma arterial embolization prognostic score; HBsAg, hepatitis virus B surface antigen; INR, international normalized ratio; MESH, model to estimate survival for hepatocellular carcinoma; mHAP, modified HAP; PSJIS, performance status combined Japan Integrated Staging system; PT, prothrombin time; TNM, tumor-node-metastasis.

endpoints of the study were 6-month and 1-year survival. Kaplan-Meier estimator survival curves and log rank tests were used to evaluate the OS rate. Likelihood ratio tests (LRTs) were used to compare different staging systems. The degree of freedom was set at 1 , so that different prognostic systems with different numbers of stages could be compared. Bias correction of Akaike information criterion (AIC) was applied. Lower AIC and higher likelihood ratio values indicate improved prognosis capacity of a staging system. Statistically significant prognostic variables in univariate analyses were identified by multivariate analysis using Cox's proportional hazards model. Receiver operating characteristic (ROC) curve analysis was performed for each staging system's predictive value for predicting 3-month, 6-month and 1-year mortality. Higher area under curve (AUC) values of the ROC curves indicate better predictive ability. Data were analyzed using SPSS (version 22.0; IBM Corp., Armonk, NY, USA) and SAS (version 9.0; SAS Institute, Inc., Cary, NC, USA). $\mathrm{P}<0.05$ was considered to indicate a statistically significant difference.
Table II. Pairwise comparison of receiver operating characteristic curves predicting 3-month survival rates.

\begin{tabular}{lllr}
\hline System 1 & System 2 & Difference & P-value \\
\hline MESH & HAP & 0.130 & 0.0008 \\
MESH & mHAP & 0.168 & 0.0001 \\
MESH & PSJIS & 0.170 & 0.0002 \\
MESH & TNM & 0.159 & $<0.0001$ \\
HAP & mHAP & 0.0373 & 0.1839 \\
HAP & PSJIS & 0.0395 & 0.4660 \\
HAP & TNM & 0.0283 & 0.5530 \\
mHAP & PSJIS & 0.00218 & 0.9706 \\
mHAP & TNM & 0.00902 & 0.8639 \\
PSJIS & TNM & 0.0112 & 0.8479 \\
\hline
\end{tabular}

HAP, hepatoma arterial embolization prognostic score; MESH, model to estimate survival for hepatocellular carcinoma; mHAP, modified HAP; PSJIS, performance status combined Japan Integrated Staging system; TNM, tumor-node-metastasis.

\section{Results}

Patient characteristics. A total of 220 patients were classified using the MESH, HAP, mHAP, TNM and PSJIS systems. The baseline characteristics of all patients studied are presented in Table I.

Staging system comparison. Analysis of the prognostic performance of the staging systems to predict 3-month, 6-month and 1-year survival rates was performed. The AUC values for predicting 3-month survival rates for MESH, HAP, mHAP, PSJIS and TNM systems were 0.858, 0.728, 0.690, 0.688 and 0.699 , respectively. Additionally, the AUC values of MESH, HAP, mHAP, PSJIS and TNM for predicting 6-month survival rates were $0.822,0.747,0.720,0.722$ and 0.715 , whereas the respective values for predicting 1-year survival rates were $0.725,0.664,0.672,0.645$ and 0.654 .

Pairwise comparison of the AUC to predict 3-month, 6-month and 1-year survival rates revealed that the MESH staging system performed optimally in predicting 3- and 6-month survival rates (Tables II and III)(Figs 1 and 2). A statistical trend was only observed when MESH was compared with mHAP in predicting 1 -year survival rate $(\mathrm{P}=0.0797$; Table IV; Fig. 3). MESH exhibited an improved performance compared with HAP, PSJIS and TNM in predicting 1-year survival rate $(\mathrm{P}<0.05)$, while mHAP performed equally well compared with PSJIS and TNM in predicting 1-year survival rate $(\mathrm{P}>0.05$; Table IV).

Additionally, the staging systems, including MESH, HAP, mHAP, TNM and PSJIS were analyzed separately, using Kaplan-Meier estimator curves (Figs. 4-8, respectively). For the analysis of the MESH system, patients were assigned to risk groups (MESH score 0-1, low risk; score 2-4, intermediate risk; score 5-6, high risk). The median survival was 3 months for the high-risk group, 27 months for the intermediate-risk group and 41 months for the low-risk group, indicating that high-risk patients had a poor survival 
Table III. Pairwise comparison of receiver operating characteristic curves predicting 6-month survival rates.

\begin{tabular}{llll}
\hline System 1 & System 2 & Difference & P-value \\
\hline MESH & HAP & 0.0752 & 0.0076 \\
MESH & mHAP & 0.102 & 0.0016 \\
MESH & PSJIS & 0.0999 & 0.0032 \\
MESH & TNM & 0.107 & 0.0002 \\
HAP & mHAP & 0.0266 & 0.2054 \\
HAP & PSJIS & 0.0247 & 0.5311 \\
HAP & TNM & 0.0320 & 0.3498 \\
mHAP & PSJIS & 0.00184 & 0.9639 \\
mHAP & TNM & 0.00541 & 0.8813 \\
PSJIS & TNM & 0.00725 & 0.8446 \\
\end{tabular}

HAP, hepatoma arterial embolization prognostic score; MESH, model to estimate survival for hepatocellular carcinoma; mHAP, modified HAP; PSJIS, performance status combined Japan Integrated Staging system; TNM, tumor-node-metastasis.

Table IV. Pairwise comparison of receiver operating characteristic curves predicting 1-year survival rates.

\begin{tabular}{llll}
\hline System 1 & System 2 & Difference & P-value \\
\hline MESH & HAP & 0.0610 & 0.0318 \\
MESH & mHAP & 0.0525 & 0.0797 \\
MESH & PSJIS & 0.0796 & 0.00116 \\
MESH & TNM & 0.0704 & 0.0189 \\
HAP & mHAP & 0.00848 & 0.6508 \\
HAP & PSJIS & 0.0186 & 0.6167 \\
HAP & TNM & 0.00943 & 0.7866 \\
mHAP & PSJIS & 0.0271 & 0.4793 \\
mHAP & TNM & 0.0179 & 0.6189 \\
PSJIS & TNM & 0.00917 & 0.7800 \\
\hline
\end{tabular}

HAP, hepatoma arterial embolization prognostic score; MESH, model to estimate survival for hepatocellular carcinoma; mHAP, modified HAP; PSJIS, performance status combined Japan Integrated Staging system; TNM, tumor-node-metastasis.

rate (8). For the analysis of the PSJIS system, patients with a score of 0-2 represented a low-risk group, whereas patients in the high-risk group had a score of 5-7 (9). The Kaplan-Meier estimator curves exhibited different prognostic strata for MESH, HAP, mHAP, PSJIS and TNM, which was statistically different (log-rank $\mathrm{P}<0.05$ in all cases). Subsequently, Kaplan-Meier estimator analysis of survival rate revealed that the MESH staging system exhibited an excellent stratified prognostic capacity.

Following comparison of the LRT $\chi^{2}$ and AIC values of the five staging systems, MESH demonstrated the highest $\chi^{2}$ and lowest AIC value, thus suggesting an improved predictive performance compared with that of the HAP, mHAP, PSJIS and TNM systems (Table V).

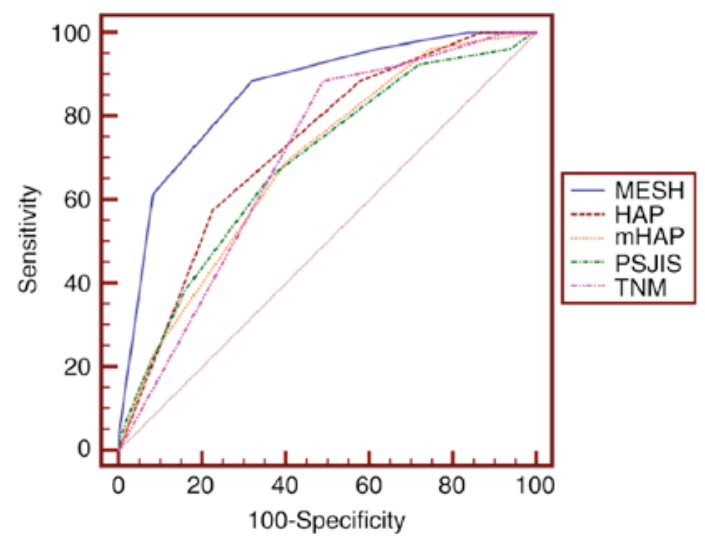

Figure 1. Receiver operating characteristic curves of MESH, HAP, mHAP, TNM and PSJIS for predicting 3-month survival. The optimal threshold value of MESH was 3 and the area under the curve of MESH was $0.858(95 \%$ confidence interval, 0.805-0.901; $\mathrm{P}<0.001)$. $\mathrm{P}<0.001$ for MESH vs. HAP, mHAP, PSJIS or TNM. MESH, model to estimate survival for hepatocellular carcinoma; HAP, hepatoma arterial embolization prognostic score; mHAP, modified HAP; PSJIS, performance status combined Japan Integrated Staging system; TNM, tumor-node-metastasis.

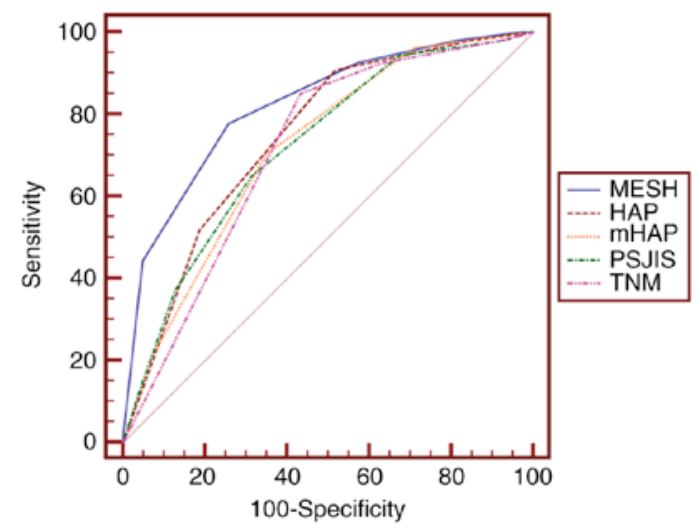

Figure 2. Receiver operating characteristic curves of MESH, HAP, mHAP, TNM and PSJIS for predicting 6-month survival. The optimal threshold value of MESH was 3 and the area under the curve of MESH was 0.822 (95\% confidence interval, 0.765-0.870; $\mathrm{P}<0.001$ ). $\mathrm{P}<0.01$ for MESH vs. HAP, mHAP, PSJIS or TNM. MESH, model to estimate survival for hepatocellular carcinoma; HAP, hepatoma arterial embolization prognostic score; mHAP, modified HAP; PSJIS, performance status combined Japan Integrated Staging system; TNM, tumor-node-metastasis.

Prognostic factors of survival in patients with HCC. Independent prognostic factors including tumor size, portal vein invasion (segmental), antiviral therapy and bilirubin for OS were revealed by univariate and multivariate analyses (Table VI).

\section{Discussion}

Many patients with $\mathrm{HCC}$ are diagnosed at an advanced stage and TACE is a major therapeutic approach. BCLC guidelines recommend patients with intermediate-stage HCC to receive TACE treatment for first-line therapy. Evidence comes from two randomized controlled clinical trials $(5,6)$. Llovet et al (5) conducted a randomized controlled trial and analyzed 112 patients. It was identified that patients with HCC undergoing chemoembolization exhibited a longer survival 
Table V. Homogeneity LRT $\chi^{2}$ test and AIC of different staging systems.

\begin{tabular}{lccc}
\hline Staging system & Homogeneity LRT $\chi^{2}$ test & AIC & P-value \\
\hline MESH & 31 & 1339 & $<0.01$ \\
PSJIS & 19 & 1354 & $<0.01$ \\
TNM & 16 & 1354 & $<0.01$ \\
mHAP & 14 & 1358 & $<0.01$ \\
HAP & 11 & 1361 & $<0.01$ \\
\hline
\end{tabular}

AIC, Akaike information criterion; HAP, hepatoma arterial embolization prognostic score; LRT, likelihood ratio test; MESH, model to estimate survival for hepatocellular carcinoma; mHAP, modified HAP; PSJIS, performance status combined Japan Integrated Staging system; TNM, tumor-node-metastasis.

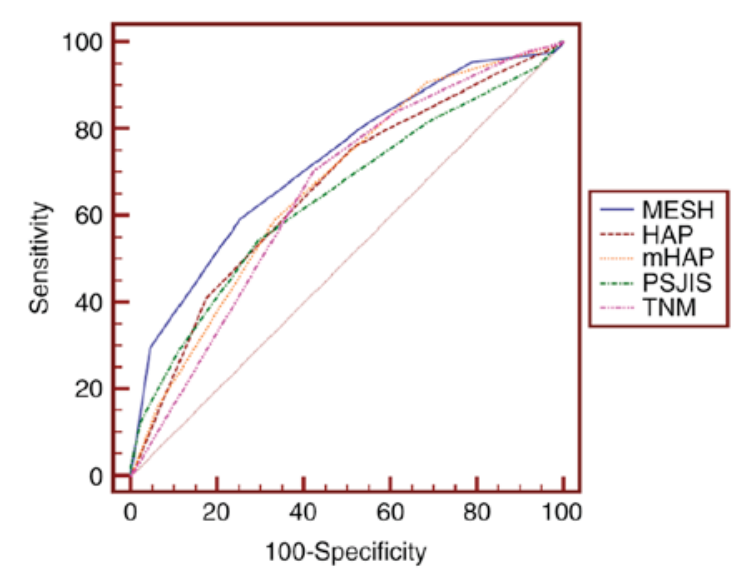

Figure 3. Receiver operating characteristic curves of MESH, HAP, mHAP, TNM and PSJIS when predicting 1-year survival. The optimal threshold value of MESH was 3 and the area under the curve of MESH was $0.725(95 \%$ confidence interval, 0.661-0.783; $\mathrm{P}<0.01) . \mathrm{P}<0.05$ for MESH vs. HAP, PSJIS or TNM. MESH, model to estimate survival for hepatocellular carcinoma; HAP, hepatoma arterial embolization prognostic score; mHAP, modified HAP; PSJIS, performance status combined Japan Integrated Staging system; TNM, tumor-node-metastasis.

time compared with patients undergoing conservative treatment. Chemoembolization decreased mortality by $53 \%$. It was concluded that under careful selection, patients with unresectable HCC received a survival benefit from chemoembolization (5). Another trial was conducted by Lo et al (6), in which 80 patients with $\mathrm{HCC}$ were examined. The survival rate of the chemoembolization group was significantly increased compared with that of the control group. Chemoembolization decreased mortality by $51 \%$. This study verified the previous results that TACE treatment significantly prolonged the survival of Asian patients with HCC at an unresectable stage.

However, it is difficult to predict which group of patients would benefit most from TACE treatment. Precise prognosis for patients with HCC under TACE treatment is needed. First, many patients may not respond to TACE although they fulfill the eligibility criteria. Furthermore, patients receiving their first course of TACE may develop liver failure and become unsuitable to receive the second embolization (10). Lastly, the development of TACE techniques has broadened the use of
TACE beyond the initial eligibility criteria, which widens the heterogeneity of the treatment group survival.

Several staging systems, including MESH, HAP, mHAP and PSJIS, have been developed for more precise prognosis for patients with HCC undergoing TACE. Liu et al (8) proposed the MESH staging system. This model was derived from the analysis of 3,182 patients with HCC from Taiwan (8), where multiple factors, including vascular invasion or metastasis, tumor size, serum $\alpha$-fetoprotein (AFP) and alkaline phosphatase (ALP) levels, were employed. MESH scores range between 0 and 6 . The authors identified that MESH improved prognostic accuracy and refined treatment strategies for patients with HCC when compared with other staging systems including BCLC, Taipei Integrated Scoring (TIS), Cancer of the Liver Italian Program (CLIP) and Hong Kong Liver Cancer (HKLC) (8). The second system, known as HAP, was established by Kadalayil et al (11) following examination of 281 patients with HCC (114 in the training set; 167 in the validation set) undergoing TACE/trans-arterial embolization (TAE). The authors employed independent prognostic factors analyzed using Cox's regression (11). Those parameters included albumin levels, tumor size, AFP levels and bilirubin levels (albumin, <36 g/dl; maximum tumor diameter, $>7 \mathrm{~cm}$; AFP, $>400 \mathrm{ng} / \mathrm{ml}$; and bilirubin, $>17 \mu \mathrm{mol} / \mathrm{l})$. Patients were categorized into HAP groups A-D. The median survival rates for HAP A, B, C and D groups were 27.6, 18.5, 9.0 and 3.6 months, respectively. Patients in the HAP C and D groups were recommended not to receive TACE owing to the poor survival rate. The HAP scoring system was further validated by Pinato et al (12) who examined 923 patients with HCC from Asia and Europe. The authors proposed a modified version of the HAP score (mHAP), based on the tumor size, albumin levels and AFP levels, but not bilirubin levels. This mHAP system was identified to offer an improved prediction of overall survival (OS) rate compared with HAP (12). Another system, the Japan Integrated Score (JIS), was established based on analysis of 722 Japanese patients with HCC (13). In addition to this system, Nishikawa et al (9) proposed the PSJIS system, which is a combination of PS with JIS and derived from 1,170 patients with HCC and with liver cirrhosis. PSJIS was identified to be an improvement over the original JIS system and the BCLC, TNM and CLIP scoring systems in predicting 1-, 3- and 5-year survival rates in patients with transcatheter arterial therapies (9).

HAP and mHAP staging systems were established based on the prognostic analysis of TACE/TAE-treated patients with HCC $(11,12)$. Patients with poor prognosis may not benefit from TACE. In the HAP staging system, patients were classified into HAP groups A-D. Median survival rates of patients classified as HAP A, B, C and D was 27.6, 18.5, 9.0 and 3.6 months, respectively (11). Patients in the HAP C and D group were advised not to receive TACE because of poor survival. The MESH score includes six common clinical variables including Child-Pugh score, vascular invasion or metastasis presence, tumor number and tumor size, PS, AFP and ALP. The MESH score considers tumor burden, serum biomarker, liver function and PS. It was demonstrated that for BCLC stage B-D patients with HCC, patients may be classified into different prognostic groups based on MESH score (8). The MESH score provided an improvement over TIS, HKLC (14) and CLIP (15).

To the best of our knowledge, MESH has not been studied in geographical areas other than Taiwan, therefore the present study 


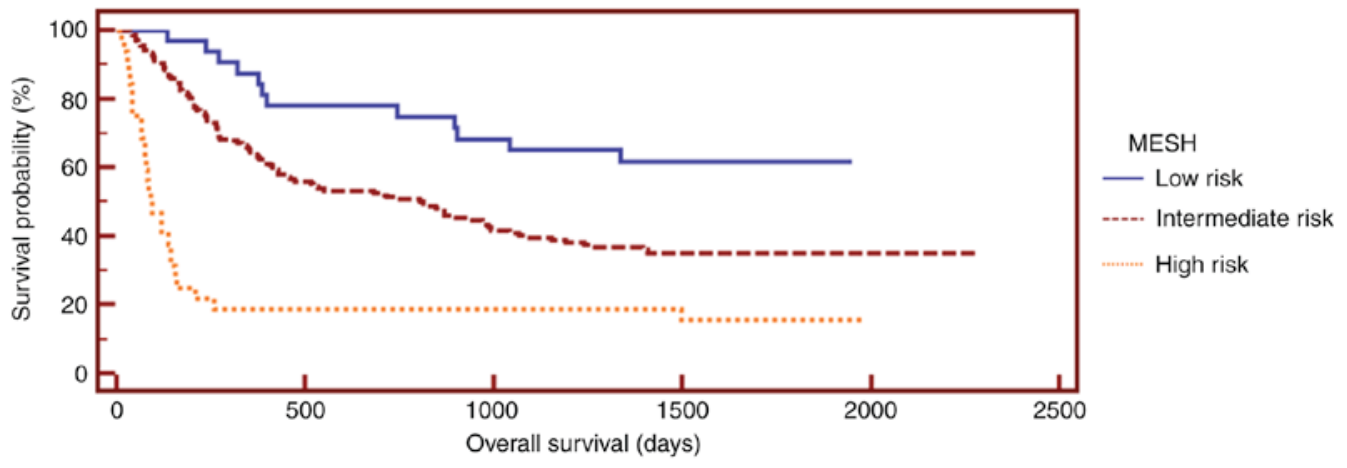

Number at risk

$\begin{array}{llllll}\begin{array}{l}\text { Group: Low risk } \\ 32\end{array} & 25 & 21 & 11 & 0 & 0 \\ \begin{array}{l}\text { Group: Intermediate risk } \\ 156\end{array} & 85 & 63 & 36 & 1 & 0 \\ \begin{array}{c}\text { Group: High risk } \\ 32\end{array} & 6 & 6 & 5 & 0 & 0\end{array}$

Figure 4. Kaplan-Meier estimator survival curves for patients with advanced HCC based on MESH score. HCC; hepatocellular carcinoma; MESH, model to estimate survival for hepatocellular carcinoma.

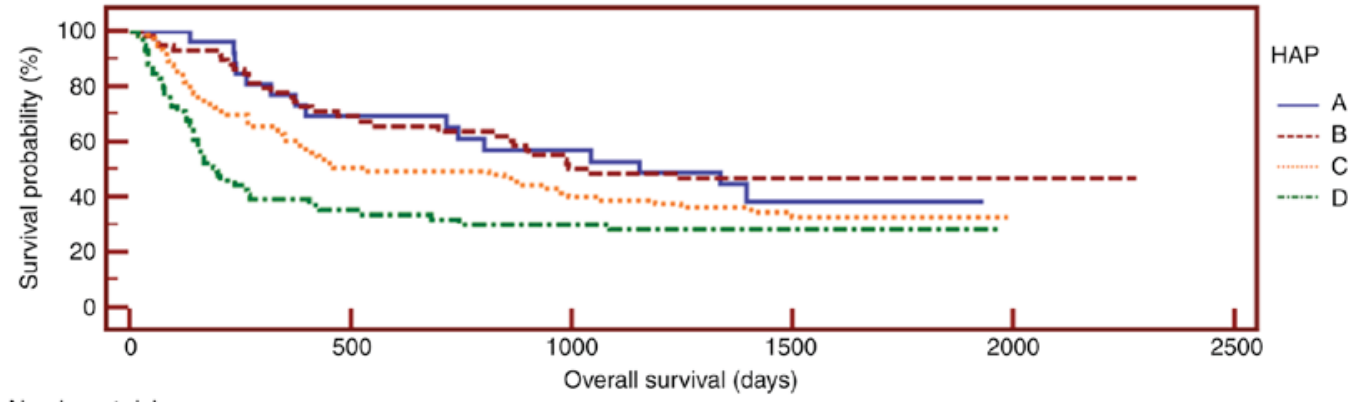

Number at risk

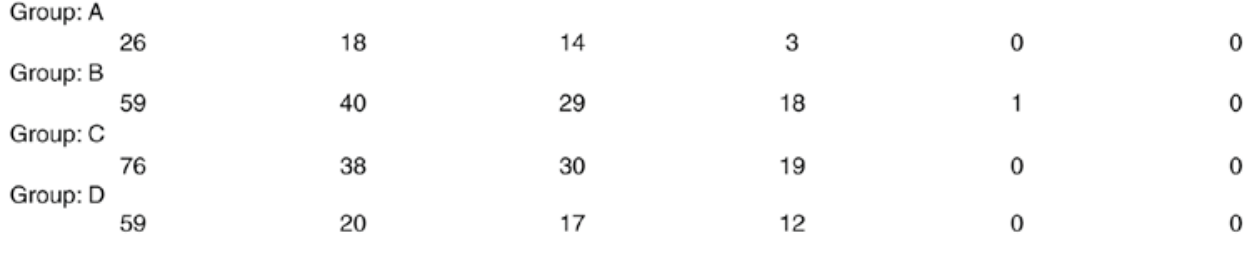

Figure 5. Kaplan-Meier estimator survival curves for patients with advanced hepatocellular carcinoma based on HAP. HAP, hepatoma arterial embolization prognostic score.

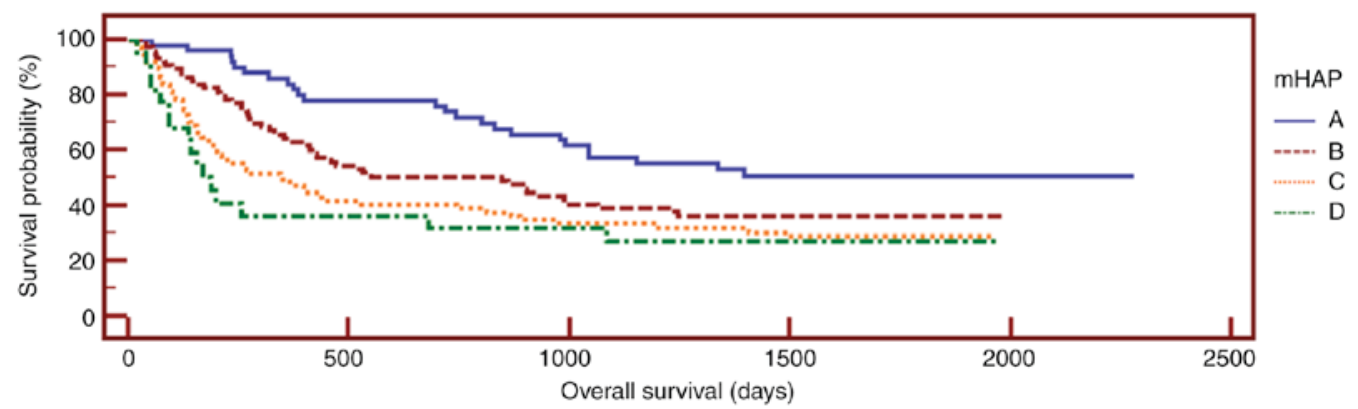

Number at risk

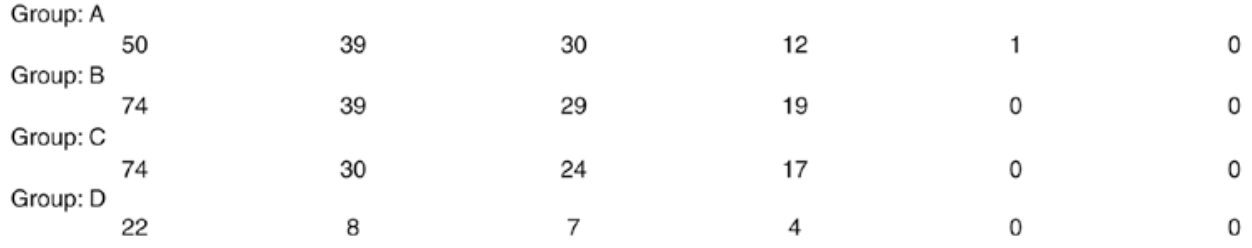

Figure 6. Kaplan-Meier estimator survival curves for patients with advanced hepatocellular carcinoma based on mHAP. mHAP, modified hepatoma arterial embolization prognostic score. 
Table VI. Univariate and multivariate analysis of prognostic factors for overall survival in 220 patients with hepatocellular carcinoma undergoing trans-arterial chemoembolization.

\begin{tabular}{|c|c|c|c|c|c|c|}
\hline \multirow[b]{2}{*}{ Variable } & \multicolumn{3}{|c|}{ Univariate analysis } & \multicolumn{3}{|c|}{ Multivariate analysis } \\
\hline & HR & $95 \% \mathrm{CI}$ & P-value & HR & $95 \% \mathrm{CI}$ & $\mathrm{P}$-value \\
\hline Sex (male/female) & 0.77 & 0.41 to 1.46 & 0.43 & & & \\
\hline Age, years $(>53 / \leq 53)$ & 0.95 & 0.68 to 1.32 & 0.75 & & & \\
\hline Tumor size ( $>50 \%$ of liver $/ \leq 50 \%$ of liver) & 2.07 & 1.48 to 2.90 & $<0.01$ & 1.64 & 1.11 to 2.42 & 0.01 \\
\hline Node status (N0/N1) & 1.99 & 1.27 to 3.11 & 0.01 & 1.24 & 0.75 to 2.04 & 0.40 \\
\hline Extrahepatic spread (yes/no) & 1.87 & 1.11 to 3.14 & 0.02 & 1.20 & 0.67 to 2.14 & 0.54 \\
\hline Portal vein invasion (segmental; yes/no) & 2.12 & 1.52 to 2.97 & $<0.01$ & 1.64 & 1.13 to 2.37 & 0.01 \\
\hline $\operatorname{AFP}(>400 / \leq 400 \mathrm{ng} / \mathrm{ml})$ & 1.41 & 1.01 to 1.97 & 0.04 & 1.03 & 0.73 to 1.48 & 0.85 \\
\hline Child-Pugh grade (A/B) & 1.32 & 0.93 to 1.88 & 0.12 & & & \\
\hline Antiviral therapy (yes/no) & 0.64 & 0.45 to 0.89 & $<0.01$ & 0.70 & 0.49 to 1.01 & 0.05 \\
\hline $\operatorname{AST}(>40 / \leq 40 \mathrm{U} / \mathrm{l})$ & 0.58 & 0.39 to 0.86 & $<0.01$ & 0.75 & 0.50 to 1.13 & 0.18 \\
\hline Bilirubin $(>51.3 / \leq 51.3 \mu \mathrm{mol} / \mathrm{l})$ & 2.22 & 1.09 to 4.54 & 0.03 & 2.54 & 1.21 to 5.36 & 0.01 \\
\hline
\end{tabular}

AFP, $\alpha$-fetoprotein; AST, aspartate transaminase; HR, hazard ratio; CI, confidence interval.
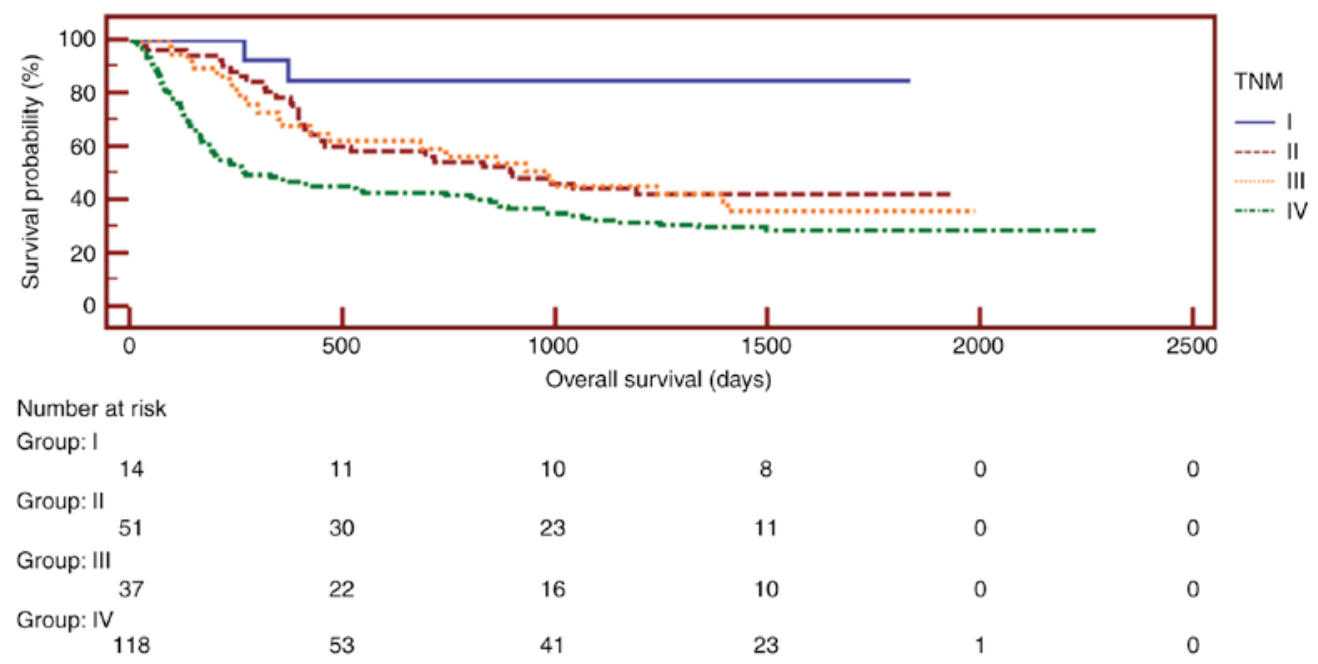

Figure 7. Kaplan-Meier estimator survival curves for patients with advanced hepatocellular carcinoma based on TNM. TNM, tumor-node-metastasis.
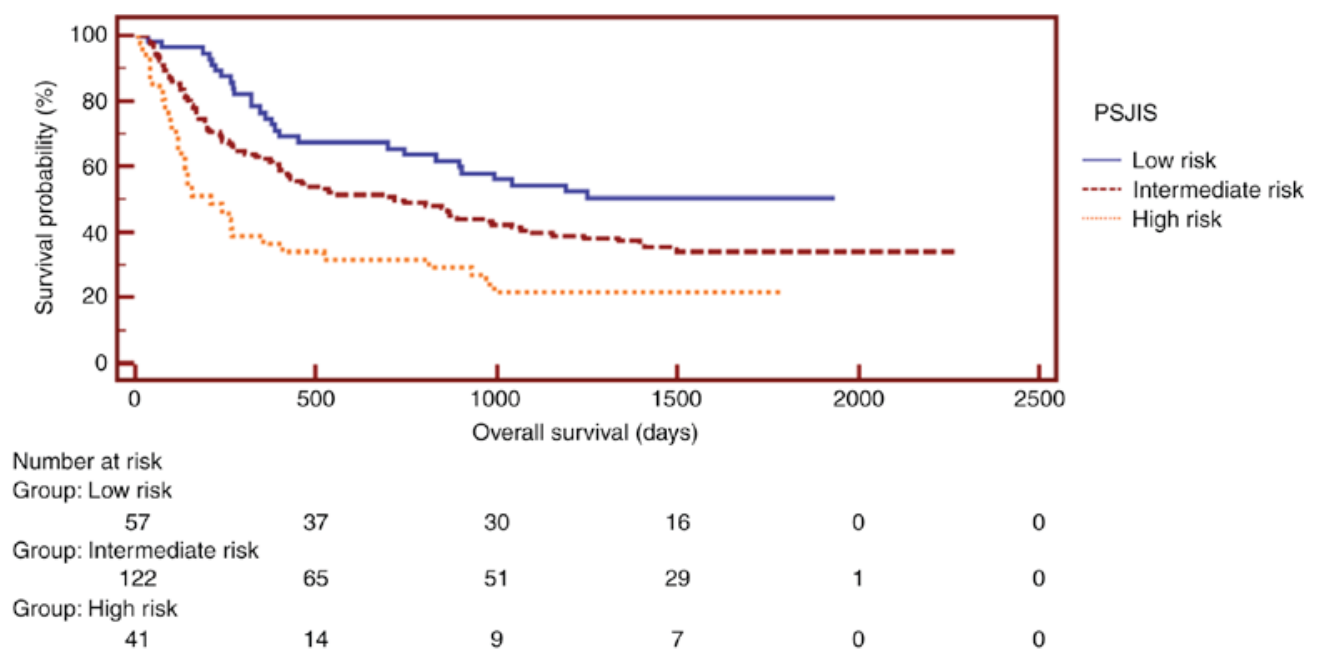

$\begin{array}{lllll}37 & 30 & 16 & 0 & 0 \\ 65 & 51 & 29 & 1 & 0 \\ 14 & 9 & 7 & 0 & 0\end{array}$

Figure 8. Kaplan-Meier estimator survival curves for patients with advanced hepatocellular carcinoma based on PSJIS. PSJIS, performance status combined Japan Integrated Staging system. 
is the first to compare MESH and other staging systems, including HAP, mHAP, PJIS and TNM in patients with HBV-associated HCC under TACE therapy. According to the results of the present study, the MESH score exhibited the highest AUC value when predicting 3-month, 6-month and 1-year survival rates. Life expectancy $>3$ months is a common inclusion criterion of TACE clinical trials. Routinely, TACE is repeated every 2-3 months (5). As for OS, MESH exhibited the highest $\chi^{2}$ value and the lowest AIC value, suggesting that MESH exhibited the optimum performance in terms of discriminatory ability, homogeneity and monotonicity. The MESH score is user-friendly and precise. The median survival of patients with HCC with a MESH score of 5-6 in the present study was 3 months. They would not benefit from TACE due to their poor survival rates.

Sub-classification of the intermediate stage of BCLC was proposed by Bolondi (16). Patients with intermediate-stage HCC were classified into four sub-classes (B1-B4) based on Child-Pugh score, tumor burden (assessed by the Milan criteria), PS and portal vein thrombosis. In the present study, the majority of patients with HCC were classified as BCLC-C, therefore Bolondi's sub-classification was not evaluated (16).

Antiviral therapy was identified as an independent prognostic factor by multivariate analyses. In the present study, all patients with HCC were associated with HBV. In total $\sim 50 \%$ of the patients received antiviral therapy. HBV reactivation and hepatic decompensation are major risks in patients with $\mathrm{HBV}$-associated HCC undergoing TACE. Previous studies have also confirmed that patients with HCC and with HBV should be considered for antiviral therapy for preventing hepatic decompensation and HCC development (17-19).

The present study has certain limitations. First, the patients included were restricted to a single center and the number of patients was limited. Additionally, the patients had HBV-associated advanced HCC. Whether the results of the present study are applicable to patients with HCC not associated with HBV is uncertain. Additional etiologies of $\mathrm{HCC}$, including $\mathrm{HCV}$ and alcohol, require further study. Therefore, standard investigations and large-scale prospective studies are required to validate the results of the present study.

In conclusion, MESH score was identified as the most accurate score system for predicting 3-month survival, 6-month survival, 1-year survival and OS rates among the five systems analyzed in the patients with HCC who received TACE treatment in the present study.

\section{Acknowledgements}

The present study was supported by National Natural Science Foundation (grant nos. 31600710 and 81372374), Natural Science Foundation of Guangdong (grant nos. 2014A030313146 and 2016A030313302) and Project on the Integration of Industry, Education and Research of Guangdong Province (grant no. 2012B091100460). The authors thank Mr. Teddy Huang (Bruker Nano Surfaces, Goleta, CA, USA) for his help in language editing.

\section{References}

1. Chen W, Zheng R, Baade PD, Zhang S, Zeng H, Bray F, Jemal A, Yu XQ and He J: Cancer statistics in China, 2015. CA Cancer J Clin 66: 115-132, 2016.
2. GBD 2015 mortality and causes of death collaborators: Global regional, and national life expectancy, all-cause mortality, and cause-specific mortality for 249 causes of death, 1980-2015: A systematic analysis for the global burden of disease study 2015 . Lancet 388: 1459-1544, 2016.

3. Bruix J and Sherman M; Practice Guidelines Committee, American Association for the Study of Liver Diseases: Management of hepatocellular carcinoma. Hepatology 42: 1208-1236, 2005.

4. European Association For Study Of Liver; European Organization For Research And Treatment Of Cancer: EASL-EORTC clinical practice guidelines: Management of hepatocellular carcinoma. Eur J Cancer 48: 599-641, 2012.

5. Llovet JM, Real MI, Montaña X, Planas R, Coll S, Aponte J, Ayuso C, Sala M, Muchart J, Solà R, et al: Arterial embolization or chemoembolization versus symptomatic treatment in patients with unresectable hepatocellular carcinoma: A randomized controlled trial. Lancet. 359: 1734-1739, 2002.

6. Lo CM, Ngan H, Tso WK, Liu CL, Lam CM, Poon RT, Fan ST and Wong J: Randomized controlled trial of transarterial lipiodol chemoembolization for unresectable hepatocellular carcinoma. Hepatology 35: 1164-1171, 2002.

7. Burrel M, Reig M,Forner A, Barrufet M, de Lope CR, Tremosini S, Ayuso C, Llovet JM, Real MI and Bruix J: Survival of patients with hepatocellular carcinoma treated by transarterial chemoembolization (TACE) using drug eluting beads. Implications for clinical practice and trial design. J Hepatol 56: 1330-1335, 2012.

8. Liu PH, Hsu CY, Hsia CY, Lee YH, Huang YH, Su CW, Lee FY, Lin HC and Huo TI: Proposal and validation of a new model to estimate survival for hepatocellular carcinoma patients. Eur J Cancer 63: 25-33, 2016.

9. Nishikawa H, Kita R, Kimura T, Endo M, Ohara Y, Sakamoto A, Saito S, Nishijima N, Nasu A, Komekado H and Osaki Y: Proposal of the performance status combined Japan integrated staging system in hepatocellular carcinoma complicated with cirrhosis. Int J Oncol 46: 2371-2379, 2015.

10. Raoul JL, Gilabert M and Piana G: How to define transarterial chemoembolization failure or refractoriness: A European perspective. Liver Cancer 3: 119-124, 2014.

11. Kadalayil L, Benini R, Pallan L, O'Beirne J, Marelli L, Yu D, Hackshaw A, Fox R, Johnson P, Burroughs AK, et al: A simple prognostic scoring system for patients receiving transarterial embolization for hepatocellular cancer. Ann Oncol 24: 2565-2570, 2013

12. Pinato DJ, Arizumi T, Allara E, Jang JW, Smirne C, Kim YW, Kudo M, Pirisi M and Sharma R: Validation of the hepatoma arterial embolization prognostic score in european and asian populations and proposed modification. Clin Gastroenterol Hepatol 13: 1204-1208, 2015.

13. Kubo S, Tanaka H, Shuto T, Takemura S, Yamamoto T, Uenishi T, Tanaka S, Hai S, Yamamoto S, Ichikawa T, et al: Prognostic effects of causative virus in hepatocellular carcinoma according to the Japan integrated staging (JIS) score. J Gastroenterol 40: 972-979, 2005.

14. Yau T, Tang VY, Yao TJ, Fan ST, Lo CM and Poon RT: Development of Hong Kong liver cancer staging system with treatment stratification for patients with hepatocellular carcinoma. Gastroenterology 146: 1691-1700, 2014.

15. Hsu CY, Huang YH, Hsia CY, Su CW, Lin HC, Loong CC, Chiou YY, Chiang JH, Lee PC, Huo TI and Lee SD: A new prognostic model for hepatocellular carcinoma based on total tumor volume: The taipei integrated scoring system. J Hepatol 53: 108-117, 2010.

16. Bolondi L, Burroughs A, Dufour JF, Galle PR, Mazzaferro V, Piscaglia F, Raoul JL and Sangro B: Heterogeneity of patients with intermediate (BCLC B) hepatocellular carcinoma: Proposal for a subclassification to facilitate treatment decisions. Semin Liver Dis 32: 348-359, 2012.

17. Li X, Zhong X, Chen ZH, Xing YF, Wu DH, Chen J, Ma XK, Lin Q, Wen JY, Wei L, et al: Hepatitis B virus DNA negativity acts as a favorable prognostic factor in hepatocellular carcinoma patients. Asian Pac J Cancer Prev 15: 9635-9641, 2014.

18. Roderburg C, Tacke F and Trautwein C: Antiviral therapy in patients with viral hepatitis and hepatocellular carcinoma: Indications and prognosis. Visc Med 32: 121-126, 2016.

19. Yu LH, Li N, Shi J, Guo WX, Wu MC and Cheng SQ: Does anti-HBV therapy benefit the prognosis of HBV-related hepatocellular carcinoma following hepatectomy? Ann Surg Oncol 21: 1010-1015, 2014. 